\title{
Is Macrophage Activation Syndrome in Kawasaki Disease Underrecognized?
}
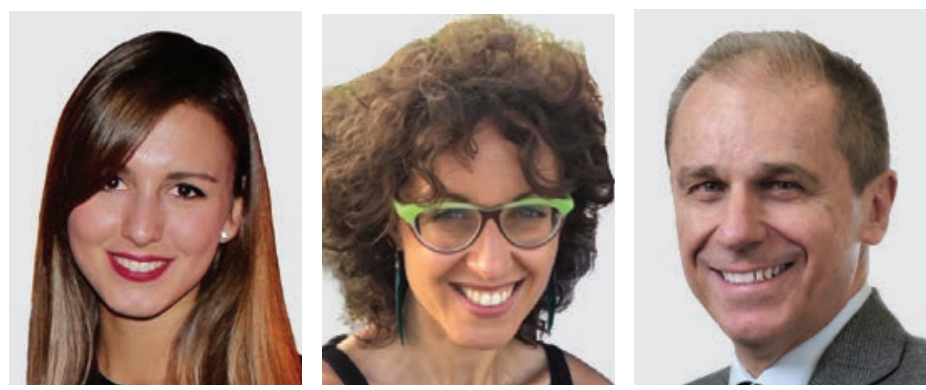

\section{Valentina Natoli ${ }^{1}$, Silvia Rosina ${ }^{2}$, and Angelo Ravelli ${ }^{3}$}

Kawasaki disease (KD) is an acute vasculitis of unknown etiology that predominantly affects children $<5$ years of age. It is now the leading cause of acquired heart disease in the pediatric age group in developed countries ${ }^{1}$. The diagnosis of classic KD is based on the presence of $\geq 5$ days of fever and the demonstration of $\geq 4$ of 5 principal clinical features: erythematous rash, bilateral nonexudative conjunctival injection, changes in the lips and oral cavity, erythema and edema in the hands and feet, and cervical lymphadenopathy, usually unilateral ${ }^{2}$. However, many children with KD, especially infants, present with fewer than 4 of the principal clinical findings (so-called incomplete $\mathrm{KD}$ ), and may experience significant delays in diagnosis. The main complication of $\mathrm{KD}$ is the development of coronary artery aneurysms, which occur in around $25 \%$ of untreated cases. High-dose intravenous immunoglobulin (IVIG) administration in the acute phase of the illness has been shown to reduce the prevalence of coronary artery abnormalities to $3-6 \%{ }^{1,3}$. However, about $10 \%$ to $20 \%$ of patients develop persistent or recurrent fever after primary therapy with IVIG and are termed IVIG resistant ${ }^{4}$. Several studies have shown that patients who are refractory to initial IVIG are at increased risk of developing coronary artery abnormalities 5 . A number of therapeutic approaches have been proposed for children who have failed to respond to initial therapy, including IVIG retreatment, corticosteroids, infliximab, cyclosporine, anakinra, plasma exchange, and cytotoxic agents ${ }^{1}$.

Macrophage activation syndrome (MAS) is a life-threatening complication of rheumatic disorders that is part of the spectrum of secondary hemophagocytic lymphohistiocytosis $(\mathrm{HLH})^{6}$. Although the pathophysiology of MAS is incompletely understood, it is thought to result from a dysfunctional immune response, which causes uncontrolled activation of the monocyte/macrophage system and ultimately leads to massive

${ }^{1}$ V. Natoli, MD, Università degli Studi di Genova, Genoa; ${ }^{2}$ S. Rosina, $M D$, PhD, IRCCS Istituto Giannina Gaslini, Genoa, Italy; ${ }^{3}$ A. Ravelli, MD, Università degli Studi di Genova, and IRCCS Istituto Giannina Gaslini, Genoa, Italy, and Sechenov First Moscow State Medical University, Moscow, Russian Federation.

Address correspondence to Prof. A. Ravelli, Clinica Pediatrica e Reumatologia, IRCCS Istituto Giannina Gaslini, Via G. Gaslini 5, 16147 Genoa, Italy. Email: angeloravelli@gaslini.org. hypersecretion of proinflammatory cytokines ${ }^{7}$. Cardinal signs and symptoms of MAS are unremitting fever, hepatosplenomegaly, neurologic dysfunction, and hemorrhagic manifestations. Typical laboratory abnormalities include a drop in blood cell lines, increased ferritin, triglycerides, liver enzymes, lactate dehydrogenase, D-dimers, and soluble CD25, and decreased fibrinogen and natural killer cell function. A characteristic histopathologic feature is the accumulation of macrophages exhibiting hemophagocytic activity in bone marrow core biopsy specimens or aspirates. If untreated, MAS may result in progressive multiorgan failure and eventual death. Timely diagnosis and prompt institution of appropriate treatment are therefore imperative.

MAS is seen most commonly in systemic juvenile idiopathic arthritis (sJIA) and in its adult counterpart, adult-onset Still disease, but is increasingly reported in patients with other rheumatic illnesses, including $\mathrm{KD}^{8,9,10,11,12,13}$. The estimated prevalence of MAS in KD is $1.1-1.9 \%^{9,11}$. However, it has been argued that MAS in KD may be more common than generally thought ${ }^{11}$. Choi, et $a l^{14}$ have suggested that a fraction of patients who are refractory to a second dose of IVIG have subclinical ("occult") MAS.

Detection of MAS in patients with KD can be challenging, because the 2 conditions share many clinical and laboratory features, including fever, rash, and elevated transaminases. Further, the frequent use of systemic glucocorticoids (GC), which are efficacious in a sizable proportion of patients with MAS, in the treatment of KD refractory to IVIG therapy ${ }^{1}$, might lead some cases of MAS presenting concurrently with KD to go unnoticed, owing to the suppression of MAS process by GC therapy. Thus, a high degree of suspicion is required for making an early diagnosis. The occurrence of MAS may affect the course and prognosis of $\mathrm{KD}$, because patients with MAS were found to have a high rate of IVIG resistance ${ }^{11,13}$. Further, a $13 \%$ mortality rate has been reported ${ }^{13}$. A comparison of the typical clinical and laboratory features of KD and MAS is presented in Table 1.

One possible explanation for the underreporting of MAS in $\mathrm{KD}$ is the lack of specific diagnostic or classification criteria. Recent studies have shown that the HLH-2004 diagnostic guidelines $^{15}$ and the 2016 classification criteria for MAS in 
Table 1. Comparison of typical clinical and laboratory features of Kawasaki disease and macrophage activation syndrome (MAS).

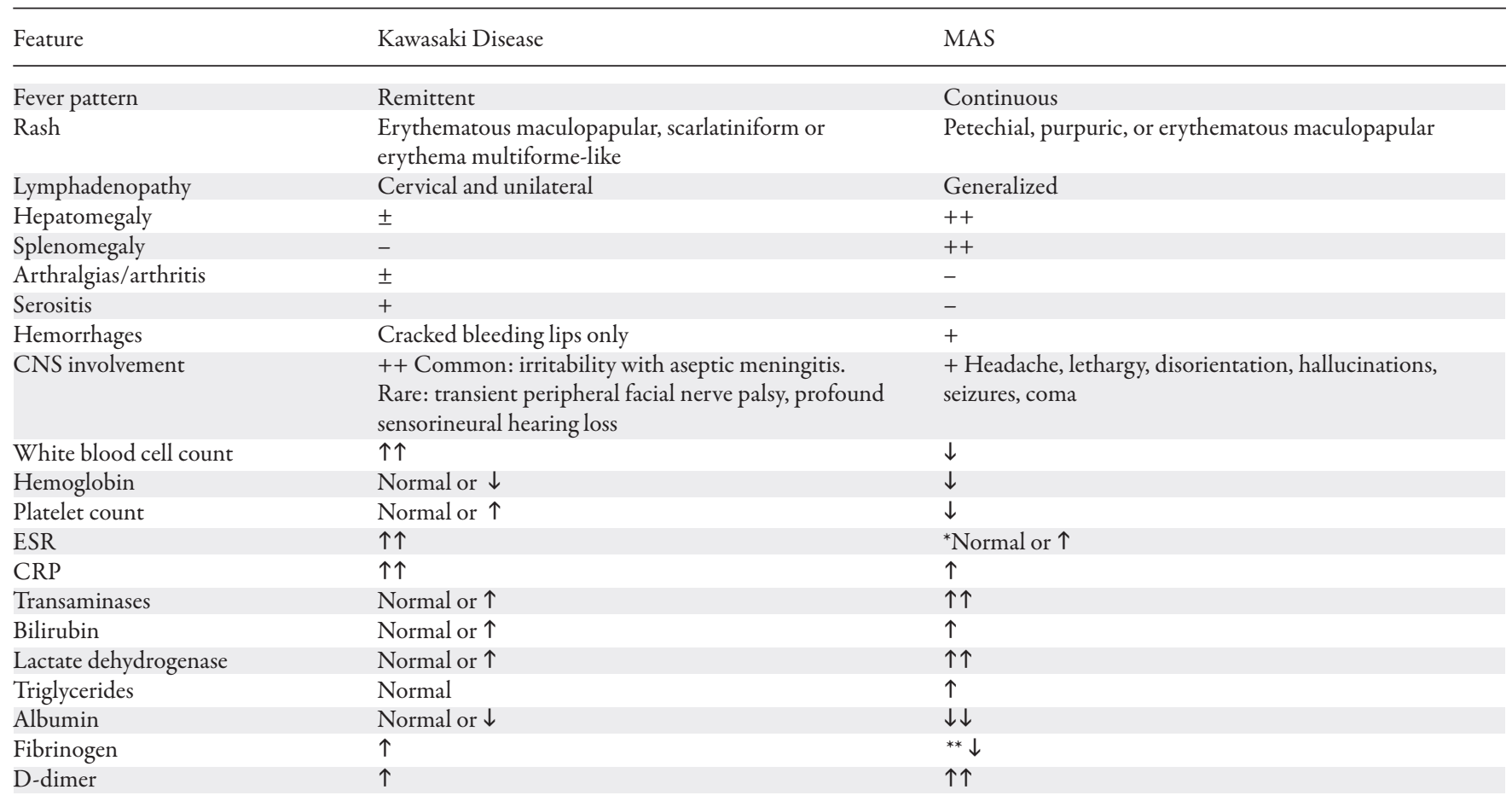

${ }^{*}$ ESR tends to decrease in MAS. ${ }^{* *}$ The absolute value of fibrinogen may be normal or still elevated in MAS; however, its decreasing trend helps with the recognition of MAS versus another inflammatory process. CNS: central nervous system; ESR: erythrocyte sedimentation rate; CRP: C-reactive protein.

sJIA ${ }^{16}$ do not have sufficient sensitivity and specificity for the detection of MAS in $\mathrm{KD}^{11,13}$. The inadequate performance of these criteria could be due to differences in MAS phenotype between KD, primary HLH and sJIA-associated MAS. To give an example, only $38 \%$ of patients with MAS in KD were found to have hypofibrinogenemia ${ }^{13}$, which is part of both HLH-2004 and 2016 sJIA-MAS criteria. Further, it is unlikely that patients with MAS in $\mathrm{KD}$, which is often associated with thrombocytosis, reach the low threshold for platelet count $\left(<100 \times 10^{9} / \mathrm{l}\right)$ requested by the HLH-2004 guidelines. In addition, the frequent occurrence of liver transaminase increase in the acute stage of $\mathrm{KD}$ makes this laboratory abnormality, which is included in the 2016 criteria for MAS in sJIA ${ }^{16}$, unsuitable for identifying MAS in KD. Note that patients with KD and MAS may not show the decrease in erythrocyte sedimentation rate (ESR) typically seen in MAS. This phenomenon has been ascribed to the neutralization of red blood count zeta potential by immunoglobulin $\mathrm{G}$ (secondary to IVIG therapy), resulting in an artificial elevation of ESR counteracting the usual process associated with MAS . These shortcomings highlight the need for a multinational collaborative effort aimed at developing classification criteria for MAS-complicating KD.

The underrecognition of MAS in the routine clinical setting could be partly due to the lack of awareness of the possible occurrence of this complication in KD. As a result, the classic laboratory markers for MAS (other than blood cell count) are not included in the routine tests for KD. Identification of the syndrome could be enhanced by recommending the inclusion of serum ferritin determination in the diagnostic blood work of all patients with $\mathrm{KD}$, particularly those with IVIG resistance. It is well known that elevated serum ferritin level is an important, available, timely, and affordable diagnostic marker of MAS. In a review of 69 cases of KD-associated MAS, the frequency of hyperferritinemia was $95 \%$ with a cutoff level $\geq 500 \mathrm{ng} / \mathrm{ml}$, as required by the HLH-2004 criteria $^{15}$, and $92 \%$ with a cutoff level of $\geq 684 \mathrm{ng} / \mathrm{ml}$, as required by the 2016 MAS-sJIA criteria ${ }^{16}$. Another alert sign for MAS is the decrease in platelet count, which contrasts with the tendency toward thrombocytosis that is typically observed in the acute stage of KD. Note that although the detection of hemophagocytosis in the bone marrow may confirm the diagnosis of MAS, this finding is frequently absent, particularly in the early stages ${ }^{9,11,13}$, and is not specific for HLH. Thus, in the presence of the classic clinical and laboratory manifestations, the diagnosis of MAS may not require the demonstration of this morphologic feature. Bone marrow aspiration might, however, be necessary in doubtful cases or to rule out a particular etiology, such as Leishmania infection.

Between March and May 2020, a rise in the number of children with some or all of the features that are seen in KD (although frequently accompanied by unusual or less common symptoms such as abdominal pain, diarrhea, and myocardial inflammation) has been noticed in some countries or country regions most seriously hit by the coronavirus disease 2019 (COVID-19) pandemic ${ }^{17,18}$. Some of these children needed urgent intensive care treatment because of the development of MAS or toxic shock-like syndrome ${ }^{19}$. Some, but not all, have 
tested positive for SARS-CoV-2. It is still unclear whether this emerging multisystem inflammatory syndrome represents true $\mathrm{KD}$ and whether it is triggered by the coronavirus. An alert has been issued by national health authorities and pediatric scientific societies to raise awareness of this condition among general practitioners and pediatricians. These observations have raised the interest of the media and are the subjects of investigation by several research groups.

It is critically important to identify MAS early, because this potentially devastating complication carries a high mortality rate and may require a more aggressive therapeutic approach than the traditional treatment protocol for KD. MAS suspicion should prompt treatment with additional IVIG and high-dose intravenous glucocorticoids. Failure to respond to this therapy should lead to consideration of additional medications used in MAS, such as cyclosporine, etoposide, or anakinra. More recently, early initiation of anakinra was found to be a rapid and effective treatment option in $\mathrm{KD}$-associated $\mathrm{MAS}^{12}$. It is noteworthy that, based on experimental findings indicating that the interleukin 1 (IL1) signaling pathway is an essential driver of disease pathogenesis, IL1 blockade with anakinra or canakinumab is being examined in clinical trials with the aim of enhancing treatment of patients with severe inflammation in the setting of acute $\mathrm{KD}^{20}$.

There is now compelling evidence that MAS in KD is underdiagnosed. Owing to the importance of hyperferritinemia for raising the suspicion of MAS, it is suggested that serum ferritin determination be included in the laboratory investigation of all patients with KD, particularly those who have persistence or recurrence of fever after the first course of IVIG. The finding of increased level of ferritin, in conjunction with other suggestive clinical and laboratory features (Table 1), should prompt the physician to diagnose MAS and to start timely and appropriate treatment. A large-scale multinational collaborative effort is required to define the characteristics of MAS in KD, to develop criteria that help practitioners in its early detection, and to establish the optimal therapeutic approach.

\section{REFERENCES}

1. McCrindle BW, Rowley AH, Newburger JW, Burns JC, Bolger AF, Gewitz M, et al. Diagnosis, treatment, and long-term management of Kawasaki disease: a scientific statement for health professionals from the American Heart Association. Circulation 2017;135:e927-e99.

2. Newburger JW, Takahashi M, Gerber MA, Gewitz MH, Tani LY, Burns JC, et al. Diagnosis, treatment, and long-term management of Kawasaki disease: a statement for health professionals from the Committee on Rheumatic Fever, Endocarditis and Kawasaki Disease, Council on Cardiovascular Disease in the Young, American Heart Association. Circulation 2004;110:2747-71.

3. Ogata S, Tremoulet AH, Sato Y, Ueda K, Shimizu C, Sun X, et al. Coronary artery outcomes among children with Kawasaki disease in the United States and Japan. Int J Cardiol 2013;168:3825-8.

4. Burns JC, Capparelli EV, Brown JA, Newburger JW, Glode MP. Intravenous gamma-globulin treatment and retreatment in Kawasaki disease. US/Canadian Kawasaki Syndrome Study Group. Pediatr Infect Dis J 1998;17:1144-8.
5. Durongpisitkul K, Soongswang J, Laohaprasitiporn D, Nana A, Prachuabmoh C, Kangkagate C. Immunoglobulin failure and retreatment in Kawasaki disease. Pediatr Cardiol 2003;24:145-8.

6. Ravelli A, Davì S, Minoia F, Martini A, Cron RQ. Macrophage activation syndrome. Hematol Oncol Clin North Am 2015; 29:927-41.

7. Ravelli A, Grom AA, Behrens EM, Cron RQ. Macrophage activation syndrome as part of systemic juvenile idiopathic arthritis: diagnosis, genetics, pathophysiology and treatment. Genes Immun 2012;13:289-98.

8. Muise A, Tallett SE, Silverman ED. Are children with Kawasaki disease and prolonged fever at risk for macrophage activation syndrome? Pediatrics 2003;112:e495.

9. Latino GA, Manlhiot C, Yeung RSM, Chahal N, McCrindle BW. Macrophage activation syndrome in the acute phase of Kawasaki disease. J Pediatr Hematol Oncol 2010;32:527-31.

10. Simonini G, Pagnini I, Innocenti L, Calabri GB, De Martino M, Cimaz R. Macrophage activation syndrome/Hemophagocytic Lymphohistiocytosis and Kawasaki disease. Pediatr Blood Cancer 2010;55:592.

11. Wang W, Gong F, Zhu W, Fu S, Zhang Q. Macrophage activation syndrome in Kawasaki disease: more common than we thought? Semin Arthritis Rheum 2015;44:405-10.

12. Shafferman A, Birmingham JD, Cron RQ. High dose anakinra for treatment of severe neonatal Kawasaki disease: a case report. Pediatr Rheumatol Online J 2014;12:26.

13. García-Pavón S, Yamazaki-Nakashimada MA, Báez M, Borjas-Aguilar KL, Murata C. Kawasaki disease complicated with macrophage activation syndrome: a systematic review. J Pediatr Hematol Oncol 2017;39:445-51.

14. Choi UY, Han SB, Lee SY, Jeong DC. Should refractory Kawasaki disease be considered occult macrophage activation syndrome? Semin Arthritis Rheum 2017;46:e17.

15. Henter JI, Horne A, Aricó M, Egeler RM, Filipovich AH, Imashuku S, et al. HLH-2004: Diagnostic and therapeutic guidelines for hemophagocytic lymphohistiocytosis. Pediatr Blood Cancer 2007; 48:124-31.

16. Ravelli A, Minoia F, Davì S, Horne A, Bovis F, Pistorio A, et al. 2016 classification criteria for macrophage activation syndrome complicating systemic juvenile idiopathic arthritis: a European League Against Rheumatism/American College of Rheumatology/ Paediatric Rheumatology International Trials Organisation collaborative initiative. Arthritis Rheumatol 2016;68:566-76.

17. Verdoni L, Mazza A, Gervasoni A, Martelli L, Ruggeri M, Ciuffreda $\mathrm{M}$, et al. An outbreak of severe Kawasaki-like disease at the Italian epicentre of the SARS-CoV-2 epidemic: an observational cohort study. Lancet 2020;395;1771-8..

18. Riphagen S, Gomez X, Gonzalez-Martinez C, Wilkinson N, Theocharis P. Hyperinflammatory shock in children during COVID-19 pandemic. Lancet 2020;395:1607-8.

19. Ma L, Zhang YY, Yu HG. Clinical manifestations of Kawasaki disease shock syndrome. Clin Pediatr 2018;57:428-35.

20. Burns JC, Koné-Paut I, Kuijpers T, Shimizu C, Tremoulet A, Arditi $\mathrm{M}$. Review: found in translation: international initiatives pursuing interleukin-1 blockade for treatment of acute Kawasaki disease. Arthritis Rheumatol 2017;69:268-76. 